\title{
Bronchogenic cyst masquerading as malignant pericardial effusion with tamponade
}

\author{
Kang-Un Choi, Byung-Jun Kim, Hong-Ju Kim, Jang Won Son, Jong-Seon Park, \\ Dong-Gu Shin, Young-Jo Kim \\ Division of Cardiology, Department of Internal Medicine, Yeungnam University College of Medicine, Daegu, Korea
}

We report on a rare case involving a 23-year-old female patient with mediastinal cystic mass complicated with acute pericarditis and cardiac tamponade. Pericardial fluid demonstrated lymphocyte-predominant exudate and the level of carcinoembryonic antigen (CEA) was unexpectedly elevated. Successive aspiration of mediastinal cystic mass revealed a very high level of CEA $(>100,000 \mathrm{U} / \mathrm{mL})$ and carbohydrate antigen $19-9(>15,000 \mathrm{ng} / \mathrm{mL})$. This patient was clinically diagnosed as an infected bronchogenic cyst complicated with pericarditis and cardiac tamponade. The treatment resulted in alleviation of her symptoms.

Keywords: Bronchogenic cyst; Pericarditis; CA19-9; CEA; Cardiac tamponade

\section{INTRODUCTION}

Most of the mediastinal cysts are asymptomatic and discovered incidentally during imaging study of the chest, however, complications including tracheal compression, superior vena cava (SVC) syndrome, atrial fibrillation, pleural effusion, and pericardial effusion have been reported [1]. Herein, we report on a case involving a young female who presented with a mediastinal cystic mass complicated with acute pericarditis and cardiac tamponade.

\section{CASE}

A 23-year-old female with no significant past medical his-

Received: September 30, 2015, Revised: October 15, 2015 Accepted: October 16, 2015

Corresponding Author: Jang Won Son, Division of Cardiology, Department of Internal Medicine, Yeungnam University College of Medicine, 170 Hyeonchung-ro, Namgu, Daegu 42415, Korea

Tel: +82-53-620-3824, Fax: +82-53-621-3310

E-mail: gubjae@yu.ac.kr tory presented to the emergency department with substernal chest pain and a high fever lasting 5 days. The chest pain worsened in the supine position and was partially relieved on sitting and in left decubitus position. On physical examination, the heart sound was decreased and both neck veins were distended.

The patient's vital signs showed blood pressure of $85 / 50$ $\mathrm{mmHg}$, heart rate of 105 beats/minute, respiratory rate of 24 breaths/minute, and body temperature of $37.6^{\circ} \mathrm{C}$. Electrocardiography showed diffuse ST segment elevation and low voltage (Fig. 1). Chest radiography showed cardiomegaly without significant pulmonary congestion or edema (Fig. 2). Laboratory investigations showed leukocytosis $14.01 \mathrm{~K} / \mu \mathrm{L}$ and neutrophils of $12.09 \mathrm{~K} / \mu \mathrm{L}$ (86.3\%); elevated high-sensitivity C-reactive protein $7.967 \mathrm{mg} / \mathrm{dL}$ (range, 0-0.06 mg/dL); normal range of cardiac biomarkers (troponin I, creatine kinase-MB) and $\mathrm{N}$-terminal prohormone of brain natriuretic peptide 223 $\mathrm{pq} / \mathrm{mL}$. Oxygen saturation while breathing room air was $96 \%$. Serum carcinoembryonic antigen (CEA) and carbohydrate antigen (CA) 19-9 level was $<0.50 \mathrm{ng} / \mathrm{mL}$ and $9.72 \mathrm{U} / \mathrm{mL}$. Chest computed tomography (CT) showed a large amount of pericardial effusion and a low density mediastinal mass measuring

Copyright (C) 2017 Yeungnam University College of Medicine

This is an Open Access article distributed under the terms of the Creative Commons Attribution Non-Commercial License (http://creativecommons.org/licenses/by-n/4.0/) which permits unrestricted non-commercial use, distribution, and reproduction in any medium, provided the original work is properly cited. 
$4.6 \times 4.8 \mathrm{~cm}$ in size between carina and left atrium (Fig. 3). Emergent bedside echocardiography showed normal left ventricular ejection fraction and no regional wall motion abnormalities. However, a large amount of pericardial effusion was detected and showed tamponade physiology (Fig. 4). Because effusion was mainly located at the posterior portion of the heart and her vital signs were unstable, emergent pericardial windows formation with pericardial biopsy was performed. The effusion was yellow colored and turbid in nature. Pericardial fluid demonstrated lymphocyte-predominant exudate and the level of glucose, protein, and lactate dehydrogenase

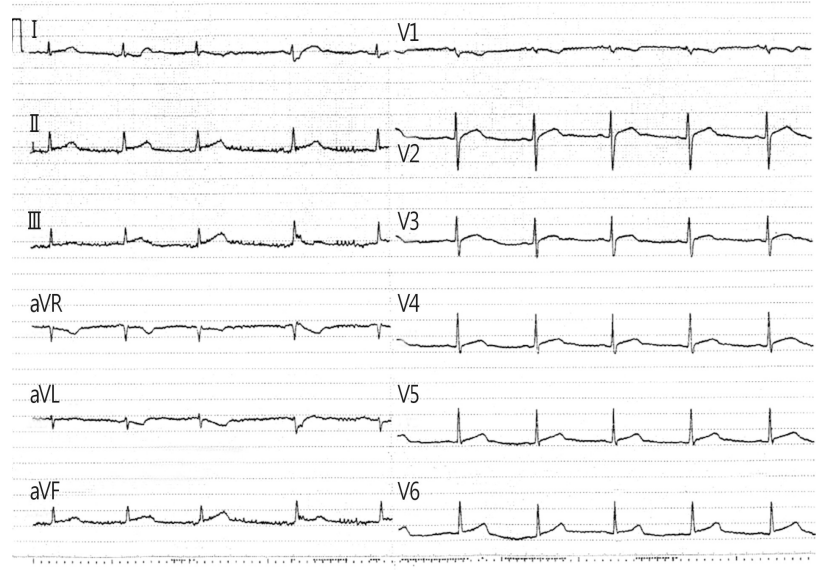

Fig. 1. Electrocardiography demonstrated diffuse ST segment elevation (precordial lead) and low voltage.

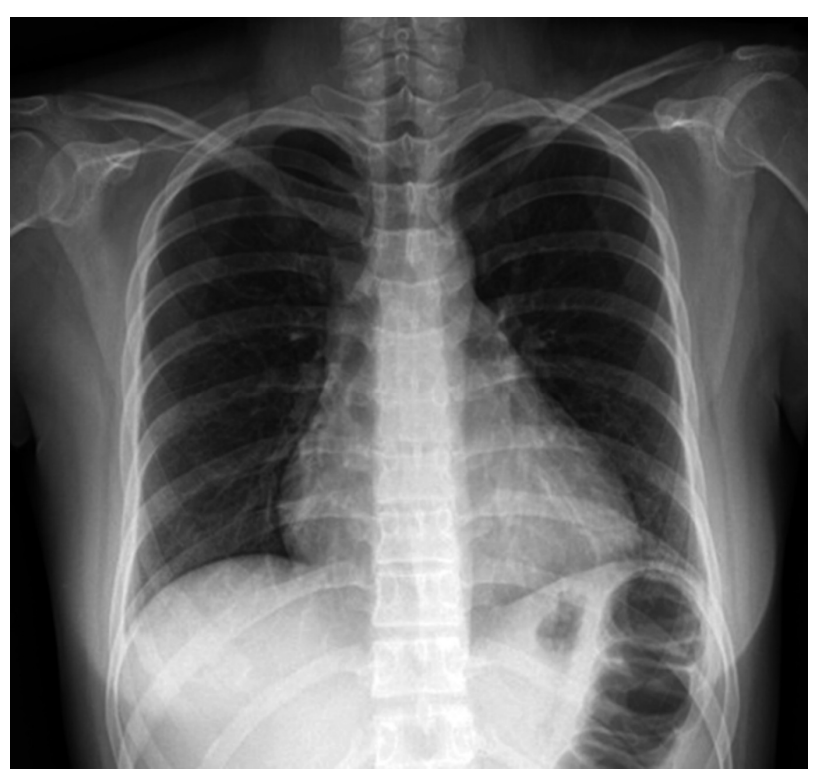

Fig. 2. Chest X-ray showed cardiomegaly without significant pulmonary congestion or edema.
(LDH) was $69 \mathrm{mg} / \mathrm{dL}, 4.86 \mathrm{~g} / \mathrm{dL}$, and 1,428 IU/L respectively. CEA level was unexpectedly elevated as $4,334 \mathrm{ng} / \mathrm{mL}$ (range, 0-10 ng/mL). All cultures and Mycobacterium tuberculosis polymerase chain reaction (MT-PCR) were negative and the cytology was reported as class I. Subsequent pathology of pericardium showed acute inflammation with reactive mesothelial hyperplasia, but no presence of malignant cells.

After the pericardial window formation, she recovered from shock, however fever was sustained (body temperature up to $38^{\circ} \mathrm{C}$ ) and chest pain worsened. A large amount of pleural effusion was newly developed and the patient complained of dyspnea. After 4 days of intravenous ceftriaxone and high dose oral ibuprofen and colchicine, her symptoms were relieved and fever subsided. Pericardial fluid drainage was also decreased to $20 \mathrm{~mL} /$ day and the pericardial tube was removed. To evaluate the cause of pericardial effusion with very high CEA level and to determine whether the mediastinal mass was malignancy or not, fusion 18F-fluorodeoxyglucose positron emission tomography (18F-FDG PET)-CT was performed (Fig. 5). On fusion PET-CT there was no 18F-FDG uptake at the mediastinal mass itself, however pericardial fluid and thickened pericardium showed significant 18F-FDG uptake
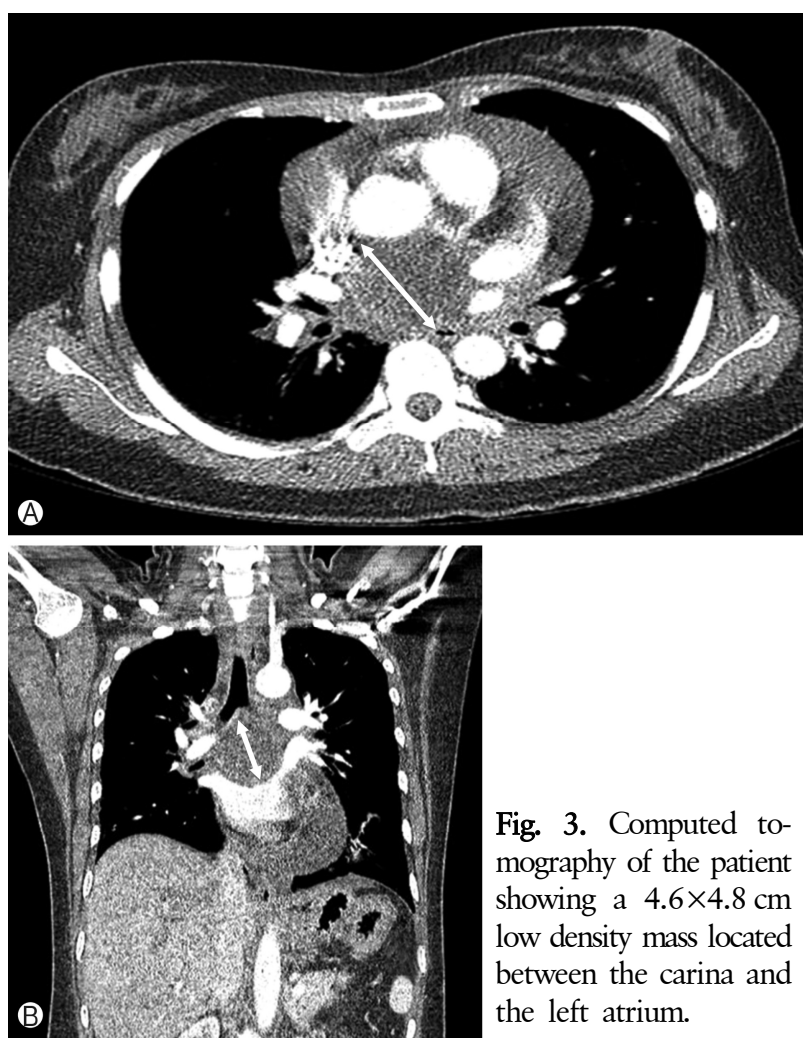

Fig. 3. Computed tomography of the patient showing a $4.6 \times 4.8 \mathrm{~cm}$ low density mass located between the carina and the left atrium. 
suggesting active inflammation. Cardiac magnetic resonance imaging (MRI) was performed for further evaluation of the

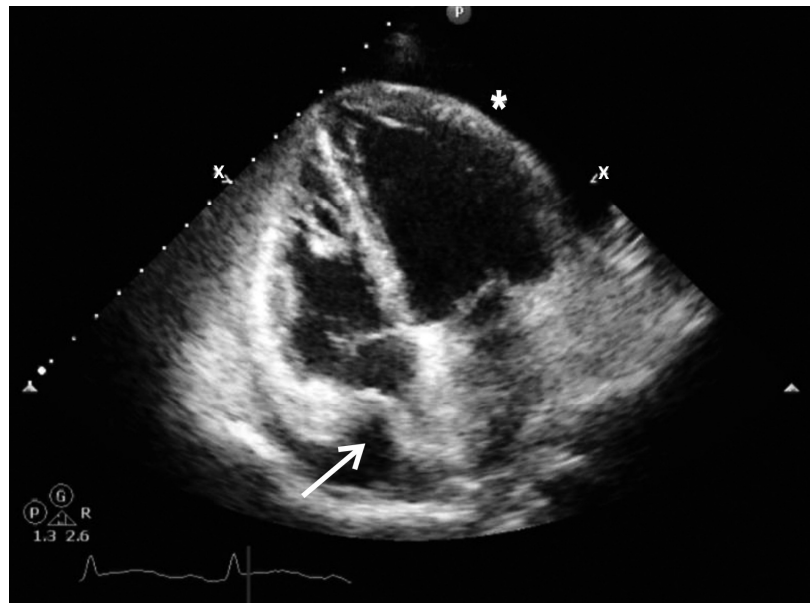

Fig. 4. Echocardiography showed right atrial systolic collapse (arrow), large amount of pericardial effusion (star).
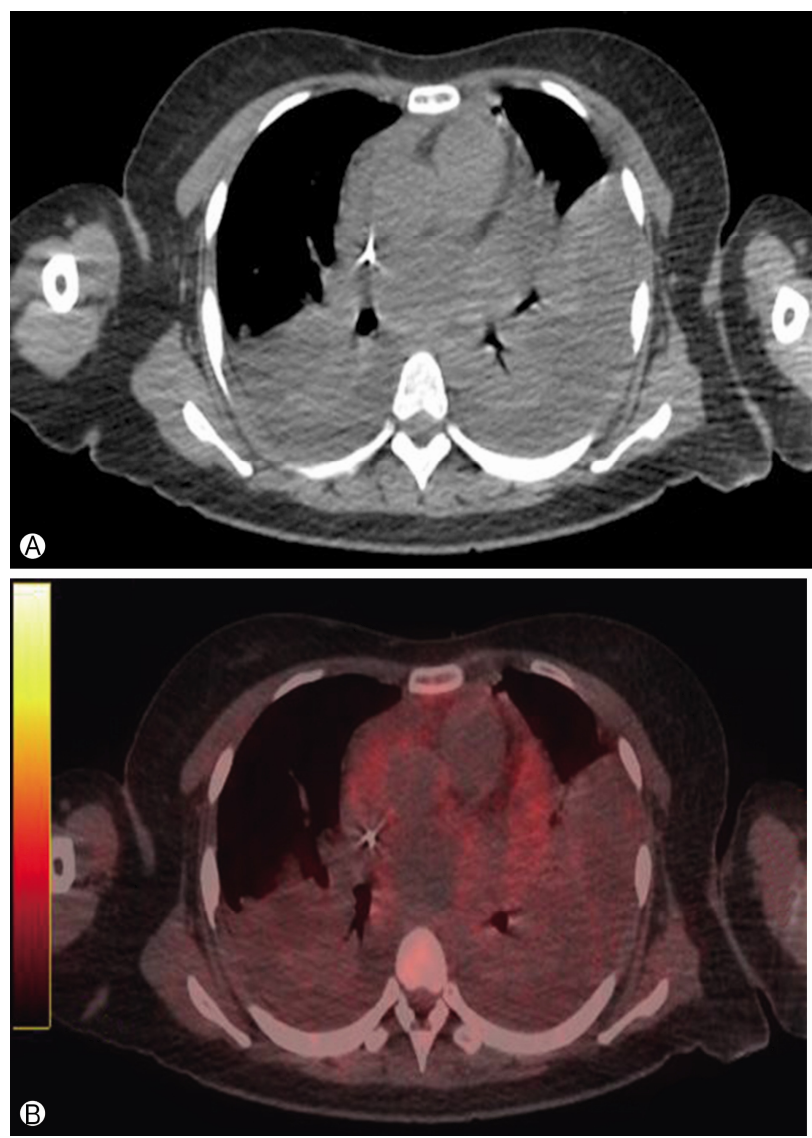

Fig. 5. 18F-fluorodeoxyglucose positron emission tomographycomputed tomography (18F-FDG PET-CT) scan shows no $18 \mathrm{~F}-$ FDG uptake at the mediastinal mass itself, and pericardium 18-F FDG uptake. mediastinal mass. On a T2 weighted image the mass showed homogeneous high signal intensity compatible with a fluid filled cyst (Fig. 6).

Because the cyst was located at just the anterior portion of the esophagus, we performed an endoscopic ultrasound (EUS) guided aspiration of the cyst without complications (Fig. 7). The cystic fluid was observed as green to brown color with a sticky nature. Fluid analysis showed polymorphonucleocytes (50\%) and other cell (50\%) exudate (total protein $5.71 \mathrm{~g} / \mathrm{dL}, \mathrm{LDH} 8,997 \mathrm{IU} / \mathrm{L})$ and a very high level of CEA $(>100,000 \mathrm{U} / \mathrm{mL})$ and CA 19-9 (>15,000 ng/mL). Adenosine deaminase level was also elevated as $86.1 \mathrm{U} / \mathrm{L}$, however acid fast bacilli stain and MT-PCR and culture were all negative. Cytology of cystic fluid was analyzed as class I.

This patient was clinically diagnosed as a bronchogenic cyst

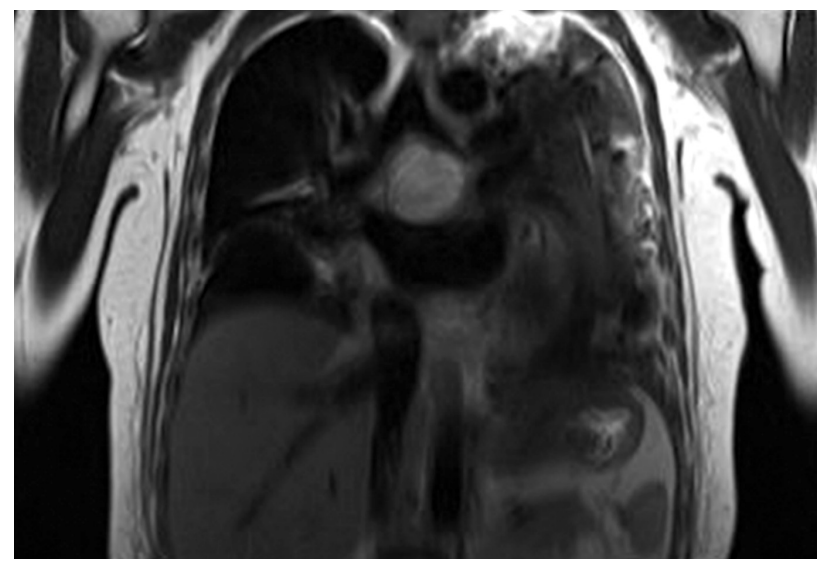

Fig. 6. Coronal T2-weighted magnetic resonance image shows a round, homogeneous, high signal intensity cystic mass with no solid component or septation.

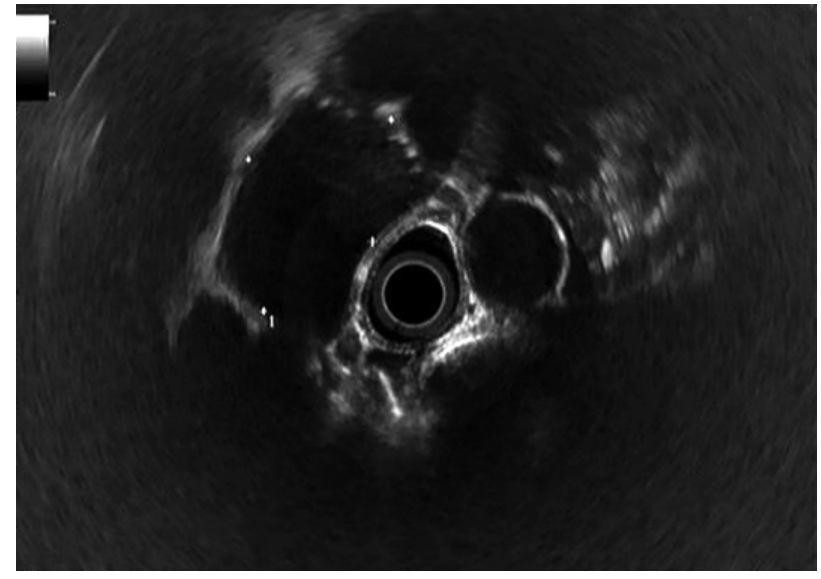

Fig. 7. Endoscopic ultrasound shows a cystic nature mass measuring $3.94 \times 2.59 \mathrm{~cm}$ in size. 
complicated with pericarditis and cardiac tamponade, and surgical removal of the cyst was recommended. However the patient refused the operation for financial reasons and was discharged. After discharge, she did not complain of any recurrent symptoms.

\section{DISCUSSION}

Mediastinal cystic mass is often discovered incidentally during imaging study of the chest and is usually asymptomatic. However when complicated by infection, hemorrhage, or rupture, a serious condition such as SVC syndrome, tracheal compression, pneumothorax, or pericardial effusion can occur [1]. In our case, the cystic mass was complicated with pericarditis and a large amount of pericardial effusion leading to life threatening cardiac tamponade.

Because of the typical nature of chest pain and febrile illness in this young female patient, we initially suspected simple viral pericarditis complicated with pericardial effusion and tamponade. However, unexpectedly elevated CEA level of pericardial fluid and mediastinal mass lesion on chest CT raised the possibility of malignant pericardial effusion [2]. Multimodality imaging tests including cardiac MRI and fusion PET-CT were performed for further evaluation of the mediastinal mass. Cardiac MRI was very helpful in verifying the nature and morphology of the mediastinal mass and showed the smooth surfaced, homogeneous cyst with no solid component or septation, which highly suggested a benign cyst of the mediastinum. No FDG uptake on fusion 18F-FDG PET-CT also suggested a benign cystic mass.

EUS guided aspiration of the cyst to clarify the discrepant results between high CEA level of pericardial fluid and imaging studies revealed a much higher level of CEA and CA 19-9 in the cystic fluid. This finding suggests that pericardial effusion with high CEA level is not a primary phenomenon, but originated from this mediastinal cyst.

Bronchogenic cysts result from abnormal budding of the tracheal diverticulum during formation of the foregut. Although rare, bronchogenic cyst is the most common benign cystic lesion of the mediastinum [3]. Although benign, there are several reports of bronchogenic cyst with high CEA and CA 19-9 level of cystic fluid [4]. Cases of high serum CEA and CA 19-9 level which were normalized after surgical resection of bronchogenic cyst were also reported by Oto et al. [5] and
Takeda et al. [6], who also demonstrated immunohistochemically that CEA and the CA 19-9 are presented within epithelial cells in cysts [5,6].

In our case, there was no elevation of CEA and CA 19-9 level in the serum; however we observed a high level of CEA and CA 19-9 in the pericardial fluid and even higher level in the mediastinal cystic fluid. By this result, we could assume that the CEA and CA 19-9 enriched bronchogenic fluid leaked to pericardium, causing pericarditis and cardiac tamponade. Yoon et al. and Sala and Coulden, who both reported on 18F-FDG PET scan of a bronchogenic cyst [7,8], showed that the bronchogenic cyst does not demonstrate FDG uptake on PET scan. In our case, there was no FDG uptake in the mediastinal cyst and this finding strongly suggested that the cyst was a benign lesion, such as bronchogenic cyst.

Although the patient refused surgical resection and the pathologic confirmation could not be made, based on comprehensive analysis of the clinical findings and multimodality imaging studies, we assumed the mediastinal cystic mass as a bronchogenic cyst complicated with acute pericarditis and cardiac tamponade.

Occasionally we encounter pericardial effusion with high CEA or CA 19-9 level, which is mainly related to malignancy. However, it can be much more complicated and differential diagnosis may be very difficult, as shown in this case. Multimodality imaging analysis including cardiac MRI and fusion 18F-FDG PET and integration of the clinical situation can be helpful for the differential diagnosis of disease in such cases.

\section{CONFLICT OF INTEREST}

No potential conflict of interest relevant to this article was reported.

\section{REFERENCES}

1. Marshall G, Cheah C, Lenzo N. Bronchogenic cyst with multiple complications. Biomed Imaging Interv J 2007;3:e42.

2. Koh KK, Kim EJ, Cho CH, Choi MJ, Cho SK, Kim SS, et al. Adenosine deaminase and carcinoembryonic antigen in pericardial effusion diagnosis, especially in suspected tuberculous pericarditis. Circulation 1994;89:2728-35.

3. Esme H, Eren S, Sezer M, Solak O. Primary mediastinal cysts: clinical evaluation and surgical results of 32 cases. Tex Heart Inst J 2011;38:371-4.

4. Uyama T, Monden Y, Sumitomo M, Miura K, Kimura S. CEA 
and CA 19-9 in benign pulmonary or mediastinal cystic lesions. J Surg Oncol 1989;41:103-8.

5. Oto T, Ando A, Aoe M, Date H, Shimizu N. Bronchogenic cyst with high serum carcinoembryonic antigen. Jpn J Thorac Cardiovasc Surg 2003;51:34-6.

6. Takeda N, Nakajima J, Yamada N, Hiroi Y, Hirata Y, Nagai R. Rupture of bronchogenic cyst in the pericardium with high carbohydrate antigen 19-9 production. Respir Med Extra 2007;
3:76-8.

7. Yoon YR, Choi J, Lee SM, Kim YJ, Cho HD, Lee JW, et al. Retroperitoneal bronchogenic cyst presenting paraadrenal tumor incidentally detected by (18)F-FDG PET/CT. Nucl Med Mol Imaging 2015;49:69-72.

8. Sala E, Coulden R. Incidental bronchogenic cyst detected on F-18 FDG positron emission tomography. Clin Nucl Med 2004; 29:494-5. 